\title{
Herramienta para la Detección de Estilos de Aprendizaje en Estudiantes utilizando la Plataforma Moodle
}

\author{
Plinio Puello, Diyina Fernández y Amaury Cabarcas \\ Universidad de Cartagena, Facultad de Ingeniería, Grupo de Investigación en Soluciones Tecnológicas de \\ Nueva Generación para el Desarrollo Social, Industrial y Empresarial, E-SOLUCIONES, Avenida del \\ Consulado, Calle 30, No. 48 - 152, Cartagena - Colombia. \\ (e-mail: ppuellom@unicartagena.edu.co, diyinafdez@gmail.com, acabarcasa@unicartagena.edu.co)
}

Recibido Mar. 10, 2014; Aceptado Abr. 14, 2014; Versión final recibida Jun. 24, 2014

\begin{abstract}
Resumen
Se ha desarrollado una herramienta para detectar los estilos de aprendizaje en estudiantes que utilizan Sistemas de Gestion del Aprendizaje (SGA) en el test de Felder y Silverman. Para la construcción del producto se utilizó la metodología de desarrollo de software por componentes y se adaptó el módulo Istest de Moodle. La herramienta permite detectar los estilos de aprendizaje que poseen los estudiantes, ubicándolos como activo-reflexivo, visual-verbal, sensitivo-intuitivo y secuencia-global de acuerdo a Felder y Silverman. La metodología fue utilizada en un grupo de estudiantes de primer semestre del programa de Ingeniería de Sistemas de la Universidad de Cartagena en Colombia. Luego de aplicarles el test se generaron listados detallados y globales de acuerdo a la escala utilizada por los mismos autores. Con la aplicación del test usando la herramienta el docente puede aplicar estrategias de aprendizaje permitiendo construir recursos y materiales idóneos adaptados a los estudiantes.
\end{abstract}

Palabras clave: ambientes de aprendizaje, estilos de aprendizajes, enseñanza, plataforma Moodle.

\section{Methodology for Detecting Learning Styles in Students using the Moodle Platform}

\begin{abstract}
A methodology for detecting learning styles in students using Learning Management System (LMS) based on Felder and Silverman test is proposed. To construct the product the methodology for software development by components was used and the Istest Moodle module was adapted. The proposed methodology can detect learning styles that students possess, placing them as active-reflective, visualverbal, sensory-intuitive and comprehensive-sequencing according to Felder and Silverman. As a case study, the methodology was tested by a group of freshmen students of the Systems Engineering program of the University of Cartagena in Colombia. With the use of this tool the instructor can apply learning strategies allowing the construction of resources and materials adapted to the student.
\end{abstract}

Keywords: learning environments, learning styles, teaching, Moodle platform 


\section{INTRODUCCIÓN}

Este artículo presenta un módulo desarrollado para la detección de estilos de aprendizaje de acuerdo a Felder y Silverman. Teniendo en cuenta que los docentes de la Universidad de Cartagena realizan el acompañamiento a las cátedras presenciales a través de medios educativos institucionales como la plataforma Moodle, existe la necesidad de implementar estrategias que permitan contribuir a disminuir la deserción estudiantil en los programas ofertados por la institución udeceista siendo de suma importancia el estudio de temáticas relacionadas con la forma en que aprenden los estudiantes de la universidad, y específicamente en el presente artículo se muestra cómo el módulo desarrollado ayuda con la detección del estilo de aprendizaje predominante en cada estudiante. Poniendo a disposición la herramienta resultante de esta investigación para futuros trabajos que, coherentemente con el estilo detectado, implementen las estrategias de aprendizaje idóneas permitiendo apropiar los conocimientos de forma natural y de esta manera contribuir a la disminución de la deserción por bajo rendimiento académico.

En la actualidad existen varios test para la medición de estilos de aprendizaje en estudiantes (Cué, 2009), discutidos y presentado por autores como Richard Felder, Linda Silverman, David Kolb, entre otros, los cuales implementaron formas de medir los estilos ajustados a diversos modelos pedagógicos evidenciado en sus trabajos de investigación (Kagan, 1966; Witkin, 1971; Grasha, 1975; Kolb, 1981). Sin embargo, existen otros estudios basados en algunos modelos anteriormente planteados y diseñan cuestionarios capaces de generar un perfil del desarrollo personal como (Harb, 1993; Honey, 1989) que buscan entender y mejorar el proceso de aprendizaje. No obstante, las investigaciones realizadas concluyen en crear categorías para coordinar los recursos o actividades y aumentar las abstracciones conceptuales en el individuo. Teniendo en cuenta que los diversos métodos son válidos, el uso de estas herramientas es escaso en la academia y en sistemas LMS (Learning Management System) como Moodle. Rubio () desarrollo un módulo para Tanto la academia como los LMS, podrían beneficiar los procesos educativos aumentando la adaptabilidad de sus cursos.

En este artículo se evidencia el desarrollo de un módulo o herramienta software que provee la posibilidad de detectar el estilo de aprendizaje en los estudiantes que utilizan Moodle. Con esto, futuros trabajos podrán seleccionar las herramientas adecuadas para una interpretación efectiva, es decir, que utilicen los recursos adaptativos acordes al estilo del estudiante. Para el desarrollo de la herramienta se utilizó la metodología de software basada en componentes ya que se pretendió desarrollar e integrar un módulo que funcionara bajo el mismo entorno como una actividad más. Los pasos que conforman esta metodología se detallan más adelante en el presente artículo. Esta investigación tuvo como resultado el desarrollo de un módulo para la detección de estilos de aprendizaje compatible con la plataforma Moodle, usando el test de Richard Felder y Linda Silverman, en el mismo se presenta los fundamentos teóricos importante para el entendimiento de la temática, la metodología utilizada y los resultados obtenidos de los criterios que evalúa el test de Felder y Silverman presentando una herramienta administrativa que apoya a los docentes que usen Moodle buscando una mayor apropiación de los conceptos adaptándolos a los estilos de aprendizaje de cada estudiante. Por último, se muestra el software propuesto integrado a la plataforma Moodle como opción del módulo actividades, aplicado a un grupo de experimentación compuesto por veintidós (22) estudiantes de la Universidad de Cartagena.

\section{FUNDAMENTOS TEÓRICOS}

\section{Ambientes Virtuales de Aprendizaje}

Al revisar el artículo de la profesora Jakeline Duarte (2003) titulado como Ambientes virtuales de aprendizaje: una aproximación conceptual, se puede verificar en la actualidad lo expuesto por ella desde aquella fecha, donde menciona que las organizaciones que pretenden desarrollar acciones educativas con sus integrantes más allá de pensar en una serie de contenidos, deben focalizar en los fundamentos y directrices didácticas y pedagógicas que la educación digitalizada exige, es decir, responder a los desafíos de la educación apoyado en las nuevas tecnologías y las comunicaciones que los ambientes virtuales de aprendizaje proveen a los docente y estudiantes de hoy.

Según Herrera (2006), un ambiente de aprendizaje constituye un espacio propicio para que los estudiantes obtengan recursos informativos y medios didácticos para interactuar y realizar actividades encaminadas a metas y propósitos educativos previamente establecidos. En ellos se distinguen cuatro elementos esenciales en un ambiente de aprendizaje: i) Un proceso de interacción o comunicación entre sujetos; ii) Un grupo de herramientas o medios de interacción; iii) Una serie de acciones reguladas relativas a ciertos contenidos; y iv) Un entorno o espacio en donde se llevan a cabo dichas actividades. 
El presente artículo muestra una herramienta que permite identificar los estilos de aprendizaje en los estudiantes a través de personalización del espacio donde se llevan a cabo las actividades de aprendizaje señaladas en el último de los elementos mencionados anteriormente, siendo todos muy importantes en el proceso motivacional y pedagógico que se desea al presentar los recursos y materiales a los estudiantes en un curso virtual o con apoyo a la presencialidad.

\section{Estilos y modelos de Aprendizaje}

Felder y Silverman (1988) conciben los estilos de aprendizaje como las preferencias que tiene un sujeto para recibir y procesar información. Este término se refiere a cómo se aprende de manera individual utilizando su propio método o conjunto de estrategias. Algunos modelos que clasifican los estilos de aprendizaje son los siguientes: de los cuadrantes cerebrales, de Felder y Silvermann, de estilos de aprendizaje de Kolb, de las Inteligencias múltiples y de programación neurolingüística.

Modelo de los Cuadrantes Cerebrales (Herrmann, 1996): Este modelo de cerebro compuesto por cuatro cuadrantes, resultan del entrecruzamiento de los hemisferio izquierdo y derecho del modelo Sperry, y de los cerebros límbico y cortical del modelo McLean. Los cuatro cuadrantes representan cuatro formas distintas de operar, de pensar, de crear, de aprender y, en suma, de convivir con el mundo.

Modelo de Felder y Silvermann (Felder, 1988): Estos autores desarrollaron un modelo de aprendizaje que llamaron "Modelo de Felder \& Silverman" el cual contempla cinco (5) dimensiones, donde dos de ellas fueron tomadas del modelo de aprendizaje de Kolb: Sensitivo-intuitivo, Visuales-verbales, Inductivodeductivo, Secuencia-global y Activo-reflexivo, donde más tarde son reducidas a cuatro (4) dimensiones.

Modelo de estilos de aprendizaje de Kolb (Kolb, 1975): David Kolb y Ronald Fry identifican dos (2) dimensiones principales del aprendizaje: la percepción y el procesamiento, obteniendo que el aprendizaje es el resultado de la forma de percibir y de cómo procesan lo que han percibido. En este modelo se plantean extremos: De estas capacidades: experiencia concreta (EC), observación reflexiva (OR), conceptualización abstracta (CA) y Experimentación activa (EA) se desprenden los cuatro estilos de aprendizaje donde Kolb crea un inventario para medir los puntos fuertes y débiles de las personas.

Modelo de las Inteligencias Múltiples (Gardner, 1999): Howard Gardner, menciona que la inteligencia no se puede medir con un número como el coeficiente intelectual. La inteligencia es la capacidad de ordenar los pensamientos y coordinarlos con las acciones, por esto Gardner plantea distintos tipos de inteligencia: Lingüística, Lógica, Espacial, Corporal, Interpersonal, Intrapersonal y Musical. Este modelo plantea distintos tipos de inteligencias: Lingüístico-verbal, Lógico-matemática, Visual-espacial, Cinestésico-corporal, Intrapersonal, Interpersonal y Musical.

Modelo de Programación Neurolingüística (Bandler, 1982): John Grinder y Richard Bandler lo llamaron visual-auditivo-kinestésico (VAK). Toma en cuenta tres (3) grandes sistemas para representar mentalmente la información, el visual, el auditivo y el kinestésico, empleando el sistema de representación visual siempre que se recuerden imágenes abstractas y concretas. El sistema de representación auditivo es el permite escuchar en nuestra mente voces, sonidos y música. Este modelo define tres (3) elementos como constituyentes claves de la conducta humana: i) Visual - Entiende el mundo tal como lo ve. Recuerda lo que ve; ii) Auditivo - Excelente conversador. Recuerda lo que oye; y iii) Kinestésico - Procesa asociando al cuerpo. Recuerda lo que hace

Se seleccionó el modelo del Felder y Silverman como base para este proyecto por su relevancia científica y por su apropiación tecnológica e influencia entre la comunidad de desarrolladores de sistemas de aprendizaje virtual. Este modelo ha sido utilizado en el desarrollo de sistemas de educación adaptativos tales como Cs388, Tangow, Lsas, Whurle, entre otros. (Stash, 2004).

\section{METODOLOGÍA}

Para el desarrollo de la herramienta presentada en este artículo se utilizó la metodología de desarrollo por componentes (Pressman, 2010), la cual describe cinco (5) fases que proporcionan una base de información necesaria para la realización y prueba del módulo para detección de estilos de aprendizaje utilizando plataforma Moodle, Con esta metodología se aprecian las características que permitieron crear la herramienta, sus componentes, modificaciones 0 adaptaciones de código al módulo LSTest, implementación del test de estilos de aprendizaje de acuerdo a Felder y Silverman y su prueba por medio de un experimento realizado para tal fin: 


\section{Fase 1: Investigación y evaluación de productos basados en componentes}

Se realizó una revisión bibliográfica sobre modelos y estilos de aprendizaje de acuerdo a los autores señalados en los fundamentos teóricos, entre estos se encuentran: Modelo de los Cuadrantes Cerebrales (Herrmann, 1996), Modelo de Felder y Silverman (Felder, 1988), Modelo de estilos de aprendizaje de Kolb (Kolb, 1975), Modelo de las Inteligencias Múltiples (Gardner, 1999), Modelo de Programación Neurolingüística (Bandler, 1982), entre otros.

La selección del modelo de Felder y Silverman se basa en la calidad y validez del instrumento que provee para la identificación del estilo de aprendizaje del estudiante. El cuestionario ha sido validado y probado (Felder, 2005), lo que proporciona el soporte y grado de confiabilidad que la mayoría de los otros modelos no tiene (Zatarain, 2011). El test de Felder y Silverman está constituido por cuarenta y cuatro (44) preguntas con dos opciones de respuesta que no generan calificaciones negativas sino que permiten organizar los grupos que responden a tendencias en el estilo de aprendizaje. Las opciones de respuesta son solo dos (2), a o b, y la tabulación se hace de acuerdo a una organización de las cuarenta y cuatro preguntas colocadas en cuatro columnas de once (11) respuestas cada una distribuidas por filas de forma ascendente. Al totalizar los valores de las columnas se podrá clasificar a los individuos de acuerdo a los estilos: activo/reflexivo, sensitivo/intuitivo, visual/verbal, secuencial/global.

En la tabla 1 se describen los test más relevantes relacionados con la identificación de los estilos de aprendizaje en los estudiantes basados en diversos autores.

Tabla 1: Comparativo de test existentes de modelos y estilos de aprendizaje

\begin{tabular}{|l|l|l|}
\hline Nombre del Test & Objetivo del Test & URL o ubicación \\
\hline $\begin{array}{l}\text { Cuestionario Honey- } \\
\text { Alonso de Estilos de } \\
\text { Aprendizaje }\end{array}$ & $\begin{array}{l}\text { Este cuestionario ha sido diseñado } \\
\text { para identificar su Estilo preferido } \\
\text { de Aprendizaje. }\end{array}$ & http://www.estilosdeaprendizaje.es/chaea/chaea.htm \\
\hline $\begin{array}{l}\text { Test de hemisferios } \\
\text { cerebrales }\end{array}$ & $\begin{array}{l}\text { Permite determinar cuál de los } \\
\text { hemisferios es más dominante. }\end{array}$ & http://es.sommer-sommer.com/test-cerebral/ \\
http://estrategiaspnl.com/test-de-hemisferios- \\
cerebrales-20-nueva-version/
\end{tabular}

\section{Características de LSTest}

El módulo LSTest permite la definición y evaluación de nuevos test de estilos de aprendizaje, basándose en el Cuestionario de Honey-Alonso sobre Estilos de Aprendizaje (CHAEA). Las funcionalidades que posee este módulo son las siguientes: 1) Permite la definir nuevos test según una sucesión de páginas que irán solicitando la información necesaria. (Parámetros propios del cuestionario CHAEA); 2) Permite exportar/importar tests a/desde un fichero en formato XML; y 3) Permite a los alumnos realizar un test.

Reportes en gráficas y tablas comparadas con la media de los alumnos del curso, con la media de todos los alumnos de la categoría a la que pertenece el curso y con la media de todos usuarios de la plataforma.

Permite a los profesores ver las respuestas a cada pregunta y los resultados de todos los alumnos del curso, obteniendo estadísticas, número de alumnos del curso en los que predomina cada estilo, puntuaciones máximas y mínimas obtenidas, número de alumnos que marcó cada respuesta posible para cada pregunta. 


\section{Fase 2: Características de integración de los componentes a Moodle}

Para la integración del componente desarrollado se tuvieron en cuenta las funcionalidades y arquitectura de la plataforma Moodle, las funcionalidades del componente son las siguientes: medición de estilos de aprendizaje, diligenciamiento de las preguntas del test, visualización de resultados y estadísticas individuales y grupales. En el diagrama de la Figura 1 se aprecian los casos de uso pertenecientes al módulo para la detección de los estilos de aprendizaje, los cuales son: diligenciar test, gestionar plataforma, agregar estilos de aprendizaje, aplicar test, visualizar resultados y generar estadísticas.
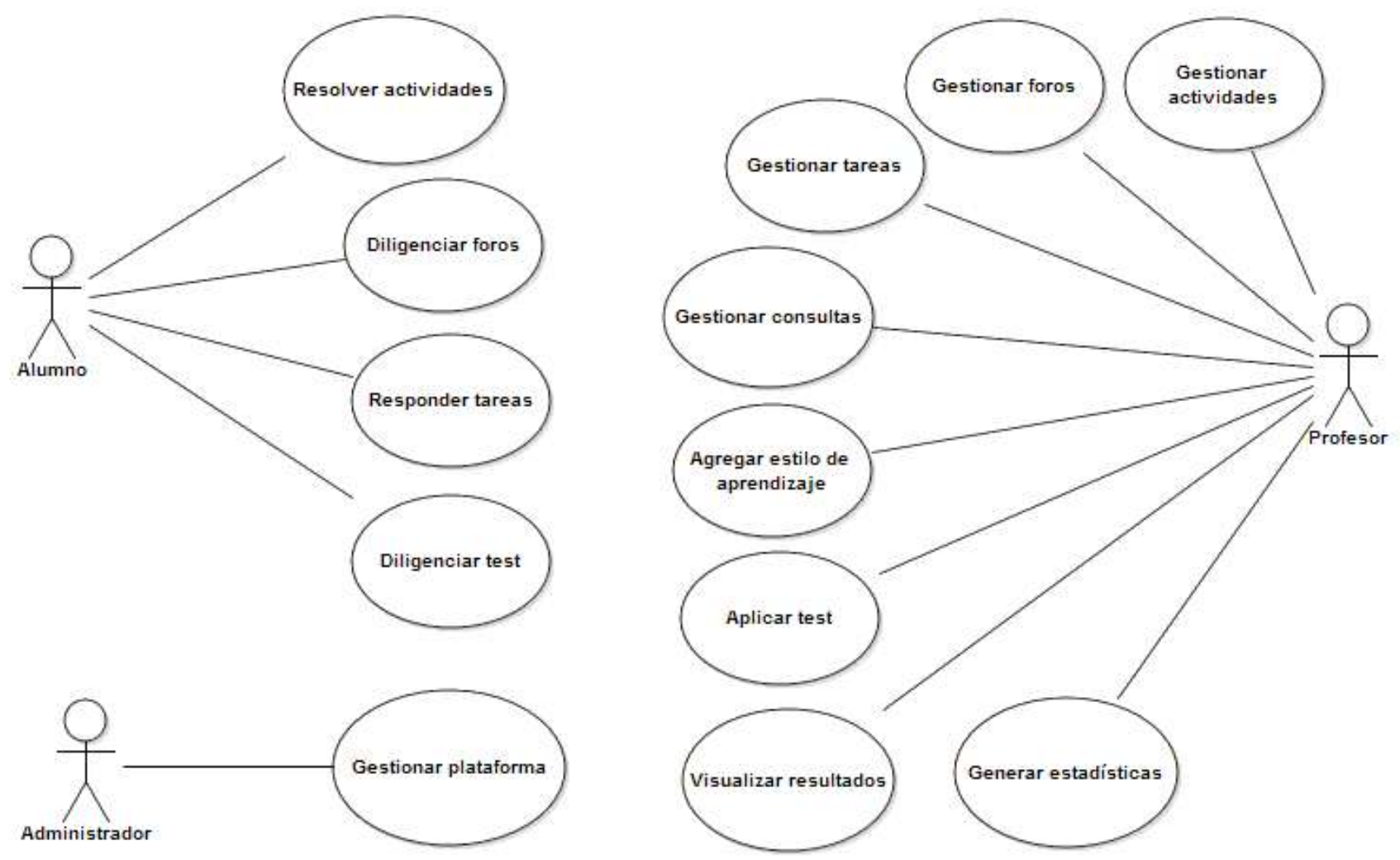

Fig. 1: Casos de uso del módulo para detección de estilos de aprendizaje

\section{Fase 3: Arquitectura del software de Moodle}

La interacción de los componentes de Moodle con el módulo para la detección de estilos de aprendizaje se aprecia en la Figura 2.

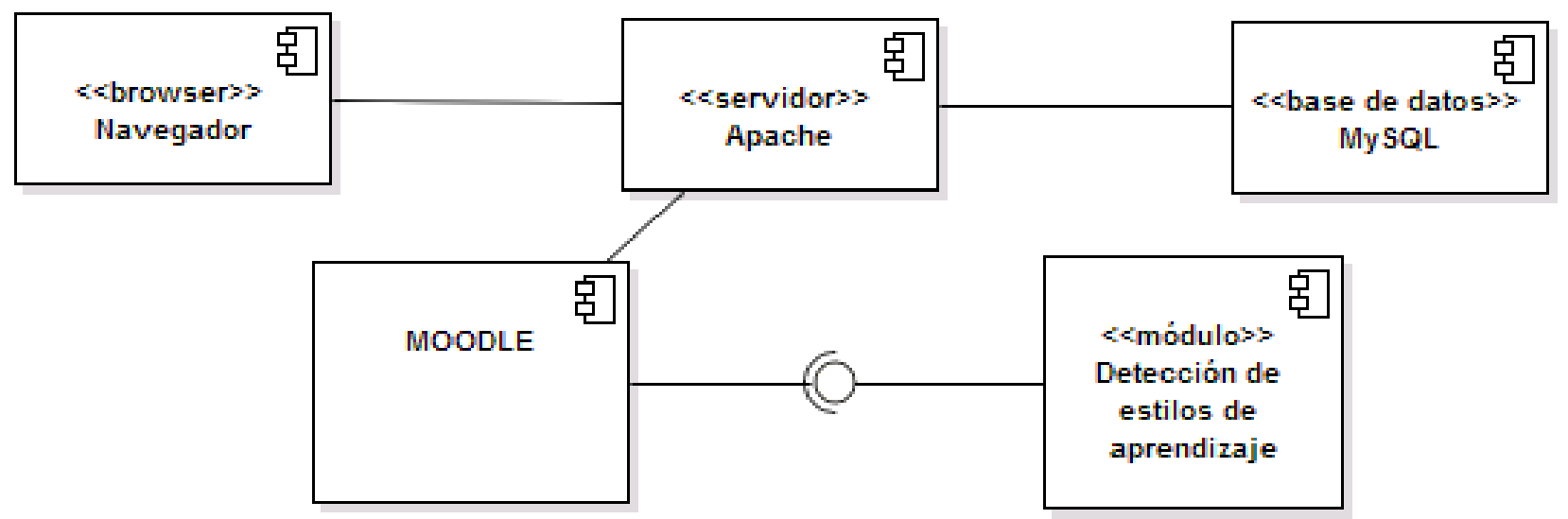

Fig. 2: Diagrama de componentes 


\section{Fase 4: Integración de componentes en la arquitectura}

El módulo funciona integrado a la base de datos de la plataforma Moodle, teniendo en cuenta que la información del usuario se encuentra previamente almacenada en ella. Se adicionaron las tablas necesarias para almacenar el histórico por estudiante y los resultados de los test realizados. Lo anterior se basó en un módulo existente llamado Istest, el cual fue modificado y adaptado al test de Felder y Silverman.

En la Figura 3 se muestra la estructura de directorios y archivos del módulo (Istest) utilizado para construir la herramienta que permitió implementar el test para la detección de estilos de aprendizaje siguiendo el Modelo de Felder y Silverman (1988).

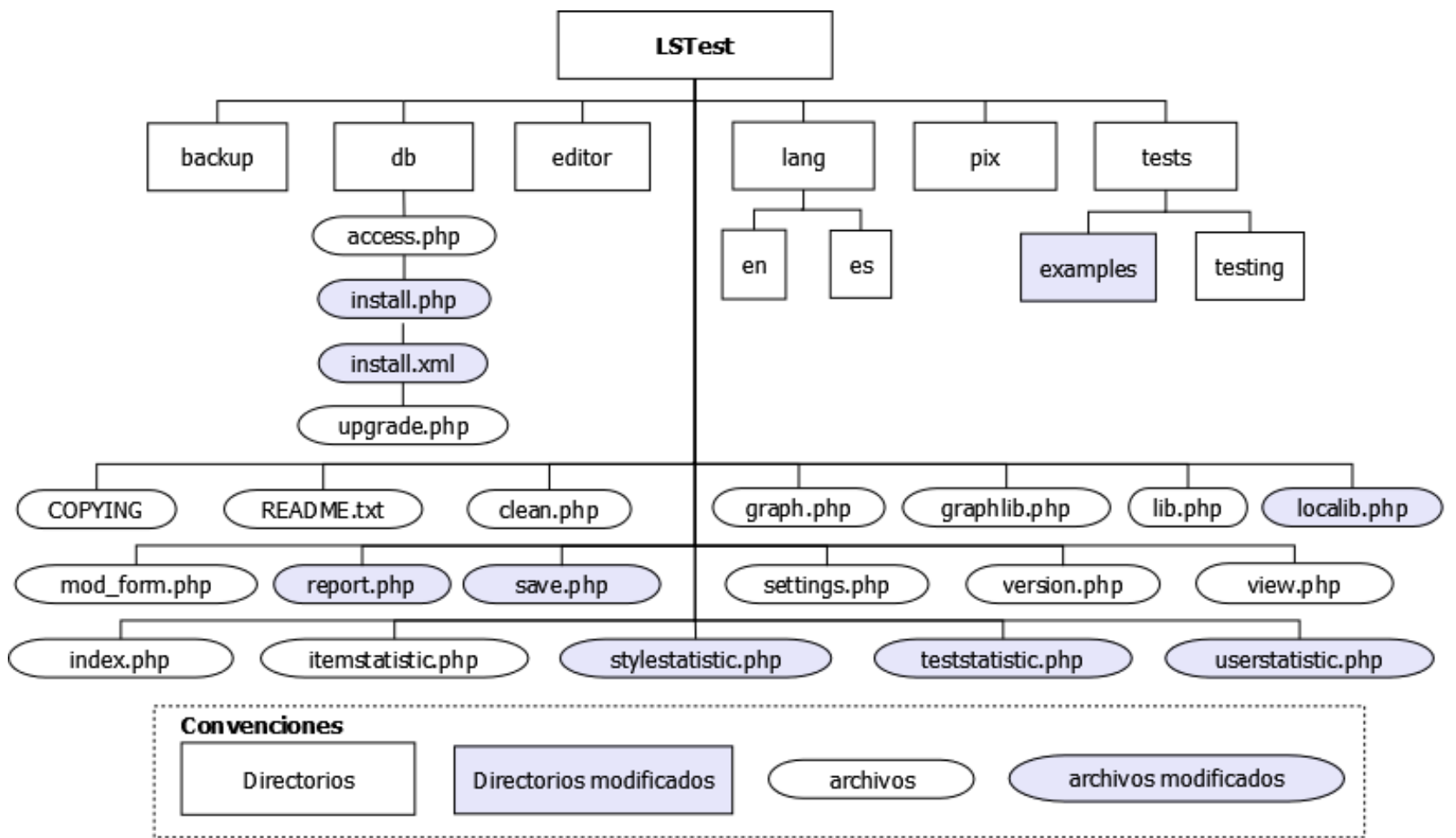

Fig. 3: Árbol de archivos módulo Istest modificado

El aporte como producto resultante de esta investigación lo constituyen las modificaciones realizadas en el código fuente del módulo LSTEST para adaptarlo al test de Felder y Silverman, las cuales se describen así:

En el directorio test->examples, se agregó el test de Felder y Silverman que tomará como test por defecto al momento de instalar el módulo en la plataforma.

Se modificaron los archivos install.php e install.xml para agregar los cambios y optimizar la instalación en el módulo como único test creado en el directorio examples.

Se agregaron y modificaron varias funciones en el archivo locallib.php, archivo donde maneja la lógica del test y de los tipos de reportes que existen en el módulo, esto con el fin de presentar los resultados acorde a las especificaciones del test de Felder y Silverman.

En el archivo save.php se modificaron algunas funciones para almacenar otras variables que permitieron organizar los resultados del test por los diferentes estilos y hacer el cálculo de los niveles en cada grupo de respuestas.

Se modificó el archivo report.php con el fin de presentar los datos acordes al test de Felder y Silverman en una hoja de cálculo por estudiante y por curso.

Se modificaron los archivos stylestatistic.php, teststatistic.php y userstatistic.php para presentar las tablas de resultados y las estadísticas acordes al test de Felder y Silverman, buscando que la presentación de éstos en la plataforma Moodle permita un mejor análisis.

Además de los aportes anteriores, el módulo fue configurado de acuerdo a las interfaces del test de Felder y Silverman, entre estas se encuentran: Estilos de aprendizaje (Activo/reflexivo, sensitivo/intuitivo, visual/verbal y secuencial/global), niveles de pertenencia de los estilos (Neutro, moderado y fuerte), umbrales de pertenencia de los estilos (ver figura 4), adición de preguntas (ver figura 6), entre otras. 


\begin{tabular}{|c|c|c|c|}
\hline \multicolumn{4}{|c|}{ Umbrales de pertenencia a los estilos $\xi$} \\
\hline \multicolumn{4}{|c|}{ Para el estilo Activo/Reflexivo } \\
\hline & $m n$ & \multicolumn{2}{|c|}{ - $\max$} \\
\hline Para el nvel Neutro: & $1 \quad-$ & -3 & $\nabla$ \\
\hline para ef nwel Moderada: & $5 \quad-$ & -7 & $\nabla$ \\
\hline Para si eivel Fuerte: & $\theta \quad-$ & -11 & - \\
\hline \multicolumn{4}{|c|}{ Para el estilo Sensitivonntuitivo } \\
\hline & $\mathrm{mn}$ & \multicolumn{2}{|c|}{$\max$} \\
\hline Pare et nivel Heutro: & $1-$ & -3 & - \\
\hline Para ef nivel Moserados. & $5 \quad-$ & .7 & - \\
\hline Pars el owvel fuerte: & $2 \quad-$ & 11 & - \\
\hline \multicolumn{4}{|c|}{ Para el estilo Visualiverbal } \\
\hline & \multicolumn{3}{|c|}{$\min -\operatorname{mar}$} \\
\hline Para ei nivel lieutro: & $1 \quad-$ & $=3$ & - \\
\hline Fara el nuvel Moderado: & $5 \quad-$ & $\therefore 7$ & $\nabla$ \\
\hline Para of anvet fuerte: & $9 \quad-$ & -11 & - \\
\hline \multicolumn{4}{|c|}{ Para el estilo Secuenciay/Giobal } \\
\hline & \multicolumn{3}{|c|}{$\min =\max$} \\
\hline Para el nivel leutro: & $1 \quad-$ & -3 & - \\
\hline Para el nuvel Moderado: & $5 \quad-$ & -7 & - \\
\hline Para el alves fuerte: & $9 \quad \div$ & -11 & 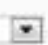 \\
\hline
\end{tabular}

Fig. 4: Umbrales de pertenencia a los estilos de aprendizaje - módulo Istest modificado

Fuente: Elaboración propia
M teat Estadisticas de alumnos Estasistican de estilos

\section{Estadisticas de alumnos (2)}

Generar informe en Eveel

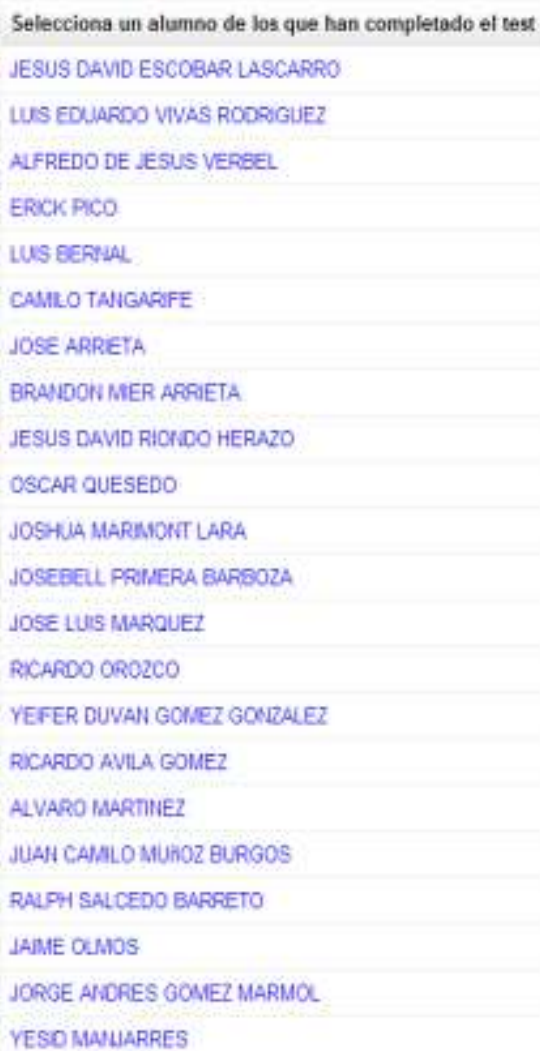

Fig. 5: Estudiantes de un asignatura del programa de Ingeniería de Sistemas Fuente: Elaboración propia

Preguntas del test $(3$

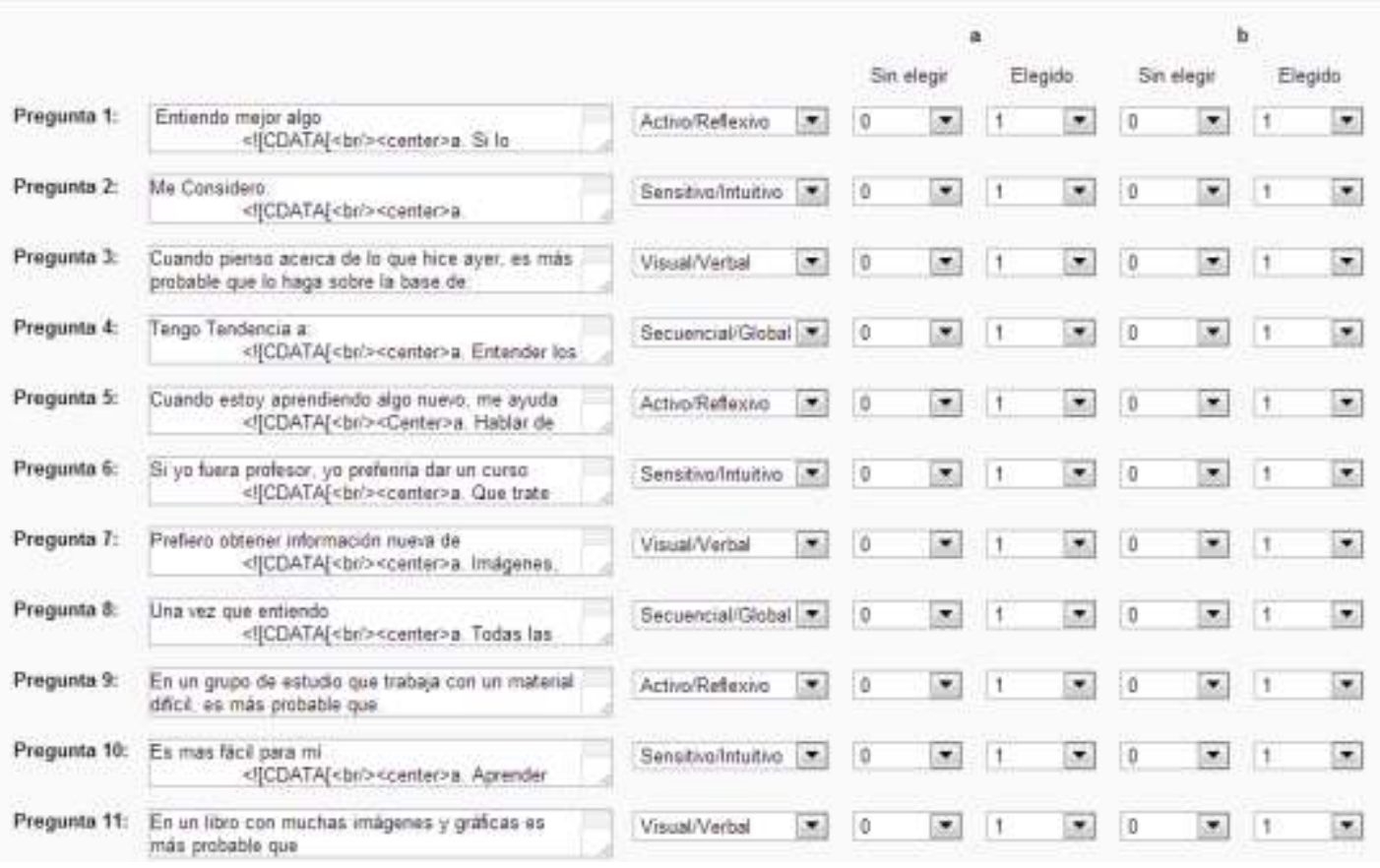

Fig. 6: Adición de preguntas del test de estilos de aprendizaje - módulo Istest modificado 


\section{Fase 5: Pruebas de funcionamiento de la herramienta}

Se realizó la instalación del módulo, verificando detalles funcionales y posibles configuraciones para mejorar sus resultados. Terminadas estas actividades, se realizó un experimento utilizando una población de veintidós (22) estudiantes del programa de Ingeniería de Sistemas de la Universidad de Cartagena. Se sustenta esta selección no probabilística de acuerdo a Fernández (2004) a través del muestreo por conveniencia el cual consiste en seleccionar unidades más convenientes para el estudio, en este caso se prioriza en aplicar este tipo de herramientas en estudiantes de primer semestre buscando su adaptación al sistema académico de la institución de educación superior udeceista y finalmente a su profesión, dando validez al muestra seleccionada para tal fin (ver figura 5).

\section{RESULTADOS Y DISCUSIÓN}

Con el fin de validar el módulo para la detección de estilos de aprendizaje bajo Moodle se logró la integración del componente desarrollado como una opción de las actividades preestablecidas en esta plataforma. Permitiendo administrar su publicación y configuración por medio de los parámetros que posee dicha herramienta. Igualmente se instaló y configuró el test de acuerdo a la escala de Felder y Silverman (Neutro de 1 a 3, Moderado de 5 a 7 y Fuerte de 9 a 11). Al aplicar el test al grupo de estudiantes seleccionados se determinó el estilo de aprendizaje de cada uno, como se aprecia en las Tablas 1 y 2. En esta última se muestra la tendencia de acuerdo al número de estudiantes y porcentaje del curso en los que predomina cada estilo.

Tabla 2: Resultado detallado del Test de Felder y Silverman

\begin{tabular}{|c|c|c|c|c|c|c|c|c|}
\hline & \multirow[t]{2}{*}{ Wient } & Estasitcas de aturnos & \multicolumn{2}{|c|}{ Lradisticas de eutilion } & \multirow[b]{3}{*}{ Vhual } & \multirow[b]{3}{*}{ Vertal } & \multirow[b]{3}{*}{ Secuencial } & \multirow[b]{3}{*}{ Gisbal } \\
\hline & & \multicolumn{3}{|c|}{ Puntaje de Estios por Usuario } & & & & \\
\hline Uneario & Actio & Reflexive & Semitive & istietivo & & & & \\
\hline ESUS UAVD ESCOAAR LASCANDO & 0 & 3 & 3 & 0 & 3 & 0 & 7 & 0 \\
\hline WUS EDUAFDO VNVAS ROOBSGLE? & 2 & e & ? & $a$ & 2 & 1 & 0 & 1 \\
\hline LLFEDO De ESUS VEREA & 6 & s & $\circ$ & ? & a & t & 1 & 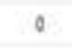 \\
\hline EFOCXPACO & y & 0 & 7 & 0 & $\rightarrow$ & 0 & + & 0 \\
\hline ULS BEFWL. & + & e & $t$ & 0 & ? & 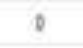 & $t$ & 0 \\
\hline CAMLO TANGAREFE & 5 & $\theta$ & 1 & $\theta$ & 3 & 0 & 0 & 5 \\
\hline SOSE ARSETA & 1 & 9 & 0 & 3 & 1 & 0 & 5 & $\theta$ \\
\hline EGADORIMER ABRETA & $t$ & $\theta$ & 0 & 1 & 3 & 0 & 0 & 1 \\
\hline IEsus OAvo scrico iesuato & , & e & 2 & 0 & 3 & 0 & 3 & 0 \\
\hline oscur suesedo & ? & 0 & 2 & 0 & 0 & + & 0 & 1 \\
\hline 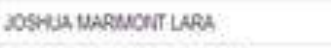 & 1 & $\theta$ & 0 & 3 & 0 & 3 & , & 0 \\
\hline DSEEEU PAMERA BAR9OLA & , & $\theta$ & 0 & 5 & 1 & $\theta$ & 0 & 6 \\
\hline SOSE LUS MAROJEL & 5 & o & 5 & 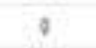 & 1 & 。 & 3 & $\theta$ \\
\hline 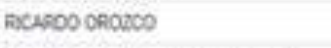 & 3 & 9 & 5 & 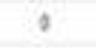 & 3 & ? & $t$ & $\theta$ \\
\hline YEFER DUNANI GCUEZ GORQUEL? & 3 & $\theta$ & $s$ & 0 & 3 & 0 & 5 & 0 \\
\hline facusoo ANR GOVEZ & 1 & $a$ & t & 0 & 1 & 0 & 3 & 0 \\
\hline NVลคO แaRT2 & 0 & 3 & 2 & 0 & 3 & 0 & $t$ & 0 \\
\hline 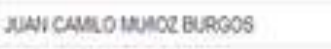 & 0 & 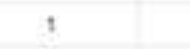 & 1 & 0 & 7 & 0 & + & 0 \\
\hline RALPH SACEDD BABRETO & 1 & 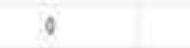 & 0 & 3 & 1 & 0 & 0 & 3 \\
\hline MUE QUVOS & 7 & e & 5 & $\theta$ & $?$ & - & 1 & 6 \\
\hline XROE ANOSES GOUEZ MUFWC & 8 & i & + & 2 & 3 & 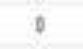 & 9 & 5. \\
\hline Yeso wuvatess & t & 0 & ? & 0 & $?$ & 0 & 3 & 0 \\
\hline
\end{tabular}

Tabla 3: Resultado grupal de tipo numérico y porcentual del Test de Felder y Silverman

\begin{tabular}{|c|c|c|c|c|c|c|c|c|c|}
\hline Alumnos en el curso & Han hecho el test & Activo & Reflexivo & Sensitivo & Intuitivo & Visual & Verbal & Secuencial & Global \\
\hline 22 & $22(100 \%)$ & $17(77 \%)$ & $5(23 \%)$ & $16(73 \%)$ & $6(27 \%)$ & $18(82 \%)$ & $4(18 \%)$ & $15(68 \%)$ & $7(32 \%)$ \\
\hline
\end{tabular}


La población estudiantil impactada inicialmente con el desarrollo del módulo basado en el test de Felder y Silverman pertenece al programa de Ingeniería de Sistemas y son aproximadamente 356 alumnos los cuales se encuentran cursando de primero a décimo semestre en el primer período académico del año 2013, permitiendo detectar los estilos de aprendizaje en los estudiantes que se encuentran matriculados en asignaturas que utilizan como apoyo a la presencialidad la plataforma Moodle, la cual es de uso institucional para los demás programas académicos udeceistas. Sin embargo, no se debe limitar al programa académico en el cual parte esta investigación sino que puede ser utilizado en estudiantes que se encuentren cursando otras áreas de formación bajo el mismo escenario de acompañamiento del aprendizaje utilizando Moodle.

La prueba de la herramienta produjo listados generales e individuales del grupo seleccionado para realizar el experimento, evidenciando la tendencia que posee cada estudiante hacia un estilo de aprendizaje de acuerdo a la clasificación de Felder y Silverman. De la figura 8 se puede identificar que el estilo predominante en el grupo de veintidós (22) estudiantes de la asignatura seleccionada para la prueba fue el estilo visual y el que menos predomina en el mismo colectivo es el estilo verbal. Lo anterior, permitirá a docentes diseñar recursos y materiales publicados en el aula virtual utilizando herramientas de aprendizaje ajustados a los estilos de los estudiantes. Los trabajos futuros que surgen de esta investigación permitirán adaptar de forma automatizada y estandarizada las herramientas de aprendizaje al estilo del estudiante por medio de la plataforma Moodle de manera similar a otros autores (Arias et al., 2008), organizando de forma preferente el listado de recursos acorde a la temática y el estilo de aprendizaje detectado en el estudiante.

La herramienta desarrollada comparada con otros test que se ofrecen por medio de páginas o enlaces externos o en ocasiones de forma escrita, es mejor debido a la facilidad de integración con Moodle, donde el estudiante asume su rol de evaluado como si se tratara de otra actividad más de la plataforma diligenciándolo de forma natural. Además presenta ventajas asociadas a la disminución de los costos relacionados con la aplicación de forma física o digital ya que se incurren en gastos de papelería y tabulación de los test escritos o infraestructura adicional como dominio y hospedaje del sitio al tenerlo publicado en páginas web externas a la misma plataforma.

\section{CONCLUSIONES}

De los resultados y el análisis del experimento realizado se pueden obtener las siguientes conclusiones: 1) Se instaló y configuró satisfactoriamente a Moodle una herramienta para la detección de estilos de aprendizaje de acuerdo a Felder y Silverman. 2) Los reportes generados permiten determinar estilos predominantes en un curso. 3) Con algunas modificaciones en el código del módulo Istest se pueden integrar otras herramientas a Moodle. 4) Es posible detectar el estilo de aprendizaje de los estudiantes en varios momentos del proceso enseñanza-aprendizaje observando posibles cambios o tendencias en ellos. 5) Al estar publicada esta herramienta en la plataforma Moodle los estudiantes adquieren las ventajas de diligenciar el test de acuerdo a su disponibilidad y momento de aprendizaje. 6) Las herramientas administrativas de Moodle permiten administrar el test como cualquier actividad o tarea incluida en la plataforma.

\section{AGRADECIMIENTOS}

Este trabajo de investigación fue financiado en el marco de la Convocatoria interna año 2011 para el fortalecimiento de Semilleros de Investigación de la Universidad de Cartagena. Los autores de la presente investigación agradecen a la Vicerrectoría de Investigaciones de la Universidad de Cartagena por el apoyo financiero asignado para el desarrollo de la misma. Además agradecemos a la especialista en el área de la Pedagogía y la Educación Superior, M.Sc.Enyel Manyoma Ledesma.

\section{REFERENCIAS}

Andrade, E., Aproximaciones a una Pedagogía del Diseño. Primer Seminario de Innovación y Competitividad, Universidad Nacional de Colombia, Facultad de Ingeniería, Bogotá - Colombia de 17, 18 y 19 de mayo, (1995).

Arias, F., D. A. Ovalle y J. Cadavid, Modelo para la Selección de Objetos de Aprendizaje Adaptados a los Estilos de los Estudiantes. GIDIA: Grupo de Investigación y Desarrollo en Inteligencia Artificial de la Escuela de Ingeniería de Sistemas de la Universidad Nacional de Colombia, (2008).

Bandler, R., y J. Grinder, Frogs into princess: Neuro linguistic programming. Royal Victorian Institute for the Blind Tertiary Resource Service, (1982). 
Cué, J. L. G., Rincón, J. A. S. y C. M. A., García, Instrumentos de medición de Estilos de Aprendizaje. Revista de estilos de aprendizaje, 4(4), 1-23 (2009).

Felder, R. M. y L. K. Silverman, Aprender y enseñar Estilos en Ingeniería de la Educación, Engr. Educación: 78(7), 674-681 (1988).

Felder, R. M. y J. Spurlin, Applications, reliability and validity of the index of learning styles. International Journal of Engineering Education, 21(1), 103-112 (2005).

Fernández, N., Investigación y técnicas de mercado. Editorial Esic, (2004).

Gardner, H., Intelligence reframed: Multiple intelligences for the twenty-first century. Basic Books, (1999).

González, O. y M. Flores, El trabajo docente: enfoques innovadores para el diseño de un curso, (1999)

Grasha, A. F. y S. W. Riechmann, Student learning styles questionnaire. University of Cincinatti, Faculty Resource Center, Cincinatti, OH, (1975).

Harb, J. N., S. O. Durrant, y R. E., Terry. Use of the Kolb learning cycle and the 4MAT system in engineering education. Journal of Engineering Education, 82(2), 70-77, (1993).

Herrera, M. A. Consideraciones para el diseño didáctico de ambientes virtuales de aprendizaje: una propuesta basada en las funciones cognitivas del aprendizaje, Revista Iberoamericana de Educación: 38(5), 1-19 (2006).

Herrmann, N., The whole brain business book, Vol. 334. New York: McGraw-Hill, (1996).

Honey, P. y A. Mumford, Learning styles questionnaire. Organization Design and Development, Incorporated, (1989).

Kagan, J., Reflection-impulsivity: The generality and dynamics of conceptual tempo. Journal of Abnormal Psychology, 71(1), 17, (1966)

Kolb, D. A., Learning styles and disciplinary differences. The modern American college, 232-255, (1981).

Kolb. D. A. y R. Fry, Toward an applied theory of experiential learning, in C. Theories of Group Process, London: John Wiley, (1975).

Oltman, P. K., E. Raskin, y H. A. Witkin, Group embedded figures test. Palo Alto, CA: Consulting Psychologists Press, (1971).

Pressman, R. S., Ingeniería del Software: Un enfoque práctico. 7ª. Edición, p.43, Editorial McGraw Hill, México, México (2010).

Stash, N. V., A. I. Cristea y P. M. De Bra,. Authoring of learning styles in adaptive hypermedia: Problems and solutions, In Proceedings of the 13th international World Wide Web conference on Alternate track papers \& posters (pp. 114-123). AC, (2004).

Zatarain Cabada, R. y M. L. Barrón Estrada, Herramienta de autor para la identificación de estilos de aprendizaje utilizando mapas auto-organizados en dispositivos móviles. Revista electrónica de investigación educativa, 13(1), 43-55 (2011). 